LBNL-

\title{
Investigation of Beam Instability Under the Effects of Long-range Transverse Wake Fields in the Berkeley Future Light Source
}

\author{
Eugene Kur and Alexander A. Zholents \\ Lawrence Berkeley National Laboratory, University of California, \\ Berkeley, California 94720
}

August 2008

\begin{abstract}
Disclaimer
This document was prepared as an account of work sponsored by the United States Government. While this document is believed to contain correct information, neither the United States Government nor any agency thereof, nor The Regents of the University of California, nor any of their employees, makes any warranty, express or implied, or assumes any legal responsibility for the accuracy, completeness, or usefulness of any information, apparatus, product, or process disclosed, or represents that its use would not infringe privately owned rights. Reference herein to any specific commercial product, process, or service by its trade name, trademark, manufacturer, or otherwise, does not necessarily constitute or imply its endorsement, recommendation, or favoring by the United states Government or any agency thereof, or The Regents of the University of California. The views and opinions of authors expressed herein do not necessarily state or reflect those of the United States Government or any agency thereof, or The Regents of the University of California.

Ernest Orlando Lawrence Berkeley National Laboratory is an equal opportunity employer.
\end{abstract}




\section{INTRODUCTION}

An ultra-relativistic charged particle bunch moving through a resonator cavity leaves behind a wake field that will affect subsequent bunches (if the bunch is not ultra-relativistic, the wake field will not be exclusively behind it). If the initial bunch enters the cavity off-axis, it will produce a transverse wake field that can then kick later bunches off the axis. Thus, even bunches that were initially traveling onaxis could be displaced and, in turn, produce their own transverse wake fields, affecting following bunches. The offsets obtained by bunches could increase along the bunch train, leading to the so-called multi-bunch beam break-up instability [1]. The purpose of our investigation is to see whether such instability will occur in the superconducting, $1.3 \mathrm{GHz}, 2.5 \mathrm{GeV}$ linac (see Table 1) planned for the Berkeley future light source (BFLS).

We assume an initial steady-state situation established for machine operation; i.e. a continuous process where every bunch follows the same trajectory through the linac, with only small deviations from the axis of the rf structures. We will look at a possible instability arising from a bunch having a small deviation from the established trajectory. Such a deviation would produce a wake field that is slightly different from the one produced by the bunches following the established trajectory. This could lead to subsequent bunches deviating further from the established trajectory. We will assume the deviations are small (at first) and so the difference in the wake field caused by a bunch not traveling along the established trajectory is well approximated by a long-range transverse dipole wake. We are concerned only with deviations from the established trajectory; thus, in our models, a transverse position of zero corresponds to the bunch traveling along the established trajectory. Under this assumption, only the additional long-range transverse dipole wake remains in our models.

\begin{tabular}{|c|c|}
\hline Type of Linac & $1.3 \mathrm{GHz}, 2.5 \mathrm{GeV}$ \\
\hline Charge per Bunch & $800 \mathrm{pC}$ \\
\hline Bunch Frequency & $1 \mathrm{MHz}$ \\
\hline Normalized Emittance & $0.5 \mu \mathrm{m}$ \\
\hline $\mathrm{E}_{0}$ & $0.241 \mathrm{GeV}$ \\
\hline $\mathrm{E}_{\text {final }}$ & $2.183 \mathrm{GeV}$ \\
\hline
\end{tabular}

Table 1: Beam and linac parameters for the Berkeley future light source 


\section{HIGHER-ORDER MODES}

When a charged bunch moves through a resonator cavity, it excites various higher-order modes (HOMS) in the cavity, which contribute to the total long-range wake. A quantity called the loss factor can be defined for each HOM; it is proportional to the amplitude of the oscillations of that HOM. Modes with higher loss factors have a greater impact on the wake field. One can usually ignore effects from HOMs with low loss factors (which HOMs can be ignored must be determined via simulation or experiment). Thus, one can find the total long-range wake using only the information on the HOMs with high loss factors.

In the present concept, the BFLS is supposed to use the same accelerator modules as those proposed in the TESLA design report [2]. Since the modules are the same, the properties of the HOMs (amplitude, frequency, damping time, etc.) are also the same, since these depend only upon the properties of the cavity. Table 2, taken from Nicoleta Baboi's thesis [3], shows which modes are considered to dominate the long-range transverse dipole wake field. We will consider only small deviations from the axis, and so the dipole wake is a good approximation for the total wake field.

\section{WAKE FIELD OF A SINGLE BUNCH}

We can calculate the transverse wake field left behind a point-like bunch (the so-called delta wake) using a formula for the wake function from Nicoleta Baboi's thesis [3]:

$$
W_{\perp}^{n \delta}(\zeta)=\sum_{m} 2 k_{\perp, m}^{n} \frac{c}{\omega_{m}} \sin \left(\omega_{m} \frac{\zeta}{c}\right) \exp \left(-\frac{\omega_{m}}{2 Q_{m}} \frac{\zeta}{c}\right), \text { for } \zeta>0
$$

where $k_{m}$ is the loss factor of the $m^{\text {th }}$ mode, $Q_{m}$ is the quality factor of the $m^{\text {th }}$ mode, $\omega_{m}$ is the frequency of the $\mathrm{m}^{\text {th }}$ mode, and $\zeta$ is the distance behind the bunch. We sum over the HOMs listed in Table 2, since they are considered to have the largest impact on the wake field. We note that the 9-cell cavities are $1 \mathrm{~m}$ in length, and thus the loss factors listed in Table 2 have units of $\mathrm{V} / \mathrm{pC} / \mathrm{m}^{3}$. The wake function thus has units of $\mathrm{V} / \mathrm{pc} / \mathrm{m}^{2}$. Defined this way, the wake function gives the transverse potential seen by a bunch, traveling over a unit length, while moving through a wake field produced by a bunch of unit charge that had an unit offset. The behavior of the dipole wake function (using dipole loss factors in the formula above) is demonstrated in Figure 1. 


\begin{tabular}{|c|c|c|c|c|c|c|}
\hline \multirow[t]{2}{*}{$\begin{array}{c}\text { Frequency } \\
\text { (simulation) } \\
{[\mathrm{GHz}]}\end{array}$} & \multicolumn{2}{|c|}{$\begin{array}{c}\text { Ave. frequency } \\
\text { (measured) } \\
{[\mathrm{GHz}]}\end{array}$} & \multirow[t]{2}{*}{$\begin{array}{c}\text { Loss factor } \\
\text { (simulation) } \\
{\left[\mathrm{V} / \mathrm{pC} / \mathrm{m}^{2}\right.} \\
/ 9 \text {-cell }]\end{array}$} & \multirow[t]{2}{*}{$\begin{array}{c}R / Q \\
\text { (simulation) } \\
{\left[\Omega / \mathrm{cm}^{2}\right.} \\
/ 9 \text {-cell }] \\
\end{array}$} & \multirow[t]{2}{*}{$\underset{\text { (meas.) }}{Q}$} & \multirow[t]{2}{*}{$\begin{array}{c}Q \\
\text { (BBU } \\
\text { limit } \\
[11]) \\
\end{array}$} \\
\hline & $\begin{array}{l}\text { 1st } \\
\text { pol. }\end{array}$ & $\begin{array}{l}\text { 2nd } \\
\text { pol. }\end{array}$ & & & & \\
\hline \multicolumn{7}{|c|}{$\mathrm{TE}_{111}$-like } \\
\hline 1.6289 & 1.6110 & 1.6114 & 0.16 & 0.01 & $1.0 \cdot 10^{6}$ & \multirow{9}{*}{$\begin{array}{l}8.4 \cdot 10^{3} \\
7.2 \cdot 10^{3} \\
7.2 \cdot 10^{3}\end{array}$} \\
\hline 1.6369 & 1.6188 & 1.6195 & 2.67 & 0.10 & $5.0 \cdot 10^{5}$ & \\
\hline 1.6506 & 1.6321 & 1.6316 & 0.02 & 0.001 & $2.0 \cdot 10^{5}$ & \\
\hline 1.6669 & 1.6500 & 1.6506 & 19.98 & 0.76 & $7.0 \cdot 10^{4 *}$ & \\
\hline 1.6888 & 1.6725 & 1.6727 & 6.05 & 0.23 & $5.0 \cdot 10^{4}$ & \\
\hline 1.7137 & 1.6978 & 1.6991 & 301.86 & 11.21 & $5.0 \cdot 10^{4 *}$ & \\
\hline 1.7383 & 1.7260 & 1.7252 & 423.41 & 15.51 & $2.0 \cdot 10^{4 *}$ & \\
\hline 1.7652 & 1.7554 & 1.7545 & 59.86 & 2.16 & $2.0 \cdot 10^{4 *}$ & \\
\hline 1.7909 & 1.7827 & 1.7831 & 49.20 & 1.75 & $7.5 \cdot 10^{3 *}$ & \\
\hline \multicolumn{7}{|c|}{ TM $_{110}$-like } \\
\hline 1.7991 & 1.7949 & 1.7948 & 21.70 & 0.77 & $1.0 \cdot 10^{4 *}$ & \multirow{9}{*}{$\begin{array}{l}4.7 \cdot 10^{4} \\
5.2 \cdot 10^{4} \\
7.6 \cdot 10^{4} \\
1.2 \cdot 10^{5} \\
1.9 \cdot 10^{5} \\
3.3 \cdot 10^{5} \\
6.7 \cdot 10^{5}\end{array}$} \\
\hline 1.8392 & 1.8342 & 1.8334 & 13.28 & 0.46 & $5.0 \cdot 10^{4 *}$ & \\
\hline 1.8531 & 1.8509 & 1.8511 & 11.26 & 0.39 & $2.5 \cdot 10^{4 *}$ & \\
\hline 1.8647 & 1.8643 & 1.8635 & 191.56 & 6.54 & $5.0 \cdot 10^{4 *}$ & \\
\hline 1.8727 & 1.8731 & 1.8732 & 255.71 & 8.69 & $7.0 \cdot 10^{4 *}$ & \\
\hline 1.8783 & 1.8795 & 1.8798 & 50.80 & 1.72 & $1.0 \cdot 10^{5 *}$ & \\
\hline 1.8820 & 1.8837 & 1.8841 & 3.17 & 0.11 & $5.0 \cdot 10^{5}$ & \\
\hline 1.8842 & 1.8864 & 1.8868 & 4.72 & 0.16 & $7.0 \cdot 10^{5}$ & \\
\hline 1.8852 & 1.8877 & 1.8881 & 2.31 & 0.01 & $1.2 \cdot 10^{6}$ & \\
\hline \multicolumn{7}{|c|}{ TE-like } \\
\hline 2.5630 & \multirow{3}{*}{\multicolumn{2}{|c|}{$\begin{array}{c}\text { no } \\
\text { statistics }\end{array}$}} & 42.41 & 1.05 & $1.0 \cdot 10^{5 *}$ & arbitr. \\
\hline 2.5704 & & & 20.05 & 0.50 & $1.0 \cdot 10^{5 *}$ & chosen \\
\hline 2.5751 & & & 961.28 & 23.80 & $5.0 \cdot 10^{4 *}$ & \\
\hline
\end{tabular}

Table 2: Dipole modes with the highest loss factors. The two independent polarization directions ( $x$ and $y$ ) have slightly different frequencies for the modes. In order to find the wake field in what we consider the $x$-direction, one needs to sum over the modes with the $1^{\text {st }}$ polarization. Similarly, summing over the modes with the $2^{\text {nd }}$ polarization will give the wake field in what we consider as the y-direction. Only data on the frequency, loss factor, and measured $Q$ was used in this investigation.

\section{FIVE RESONATOR MODEL}

We will look at a model of the acceleration system in the Berkeley FLS. The actual system has five resonator cavities, with different Twiss functions and beam energies. We make our model slightly simpler by assuming the Twiss functions are constant in each resonator, and only change between the resonators. We look at the bunches starting at a point with a betatron phase $\pi / 2$ less than the phase at the center of the first resonator. We assume that all of the bunches move on-axis (and with no angle) through that point, until one bunch starts there with an angle. For the long-range transverse dipole wake field, a bunch offset in the x-direction will leave behind a wake field that deflects only in the $\mathrm{x}$ direction. We can thus look at the $\mathrm{x}$ - and $\mathrm{y}$-directions separately. We will assume that the off-angle bunch (which we will index by the number 0 ) has an angle of $\sigma_{\mathrm{x}}{ }^{\prime}$ ( or $\left.\sigma_{\mathrm{y}}{ }^{\prime}\right)$. This setup is shown in Figure 2. 


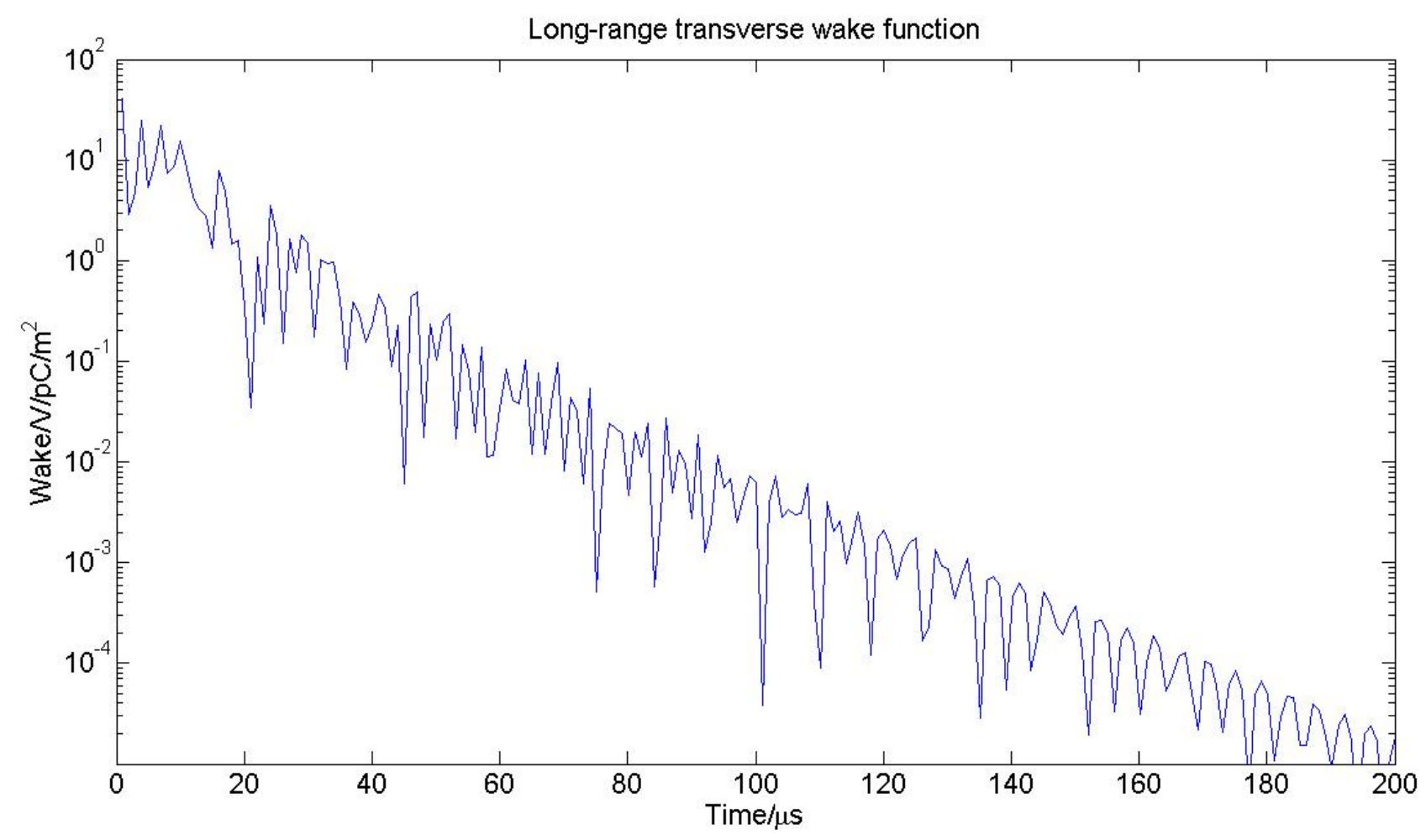

Figure 1: Long-range transverse dipole wake function for a point-like bunch moving through a resonant cavity. The wake field for a point-like bunch (represented above) is known as a delta wake.

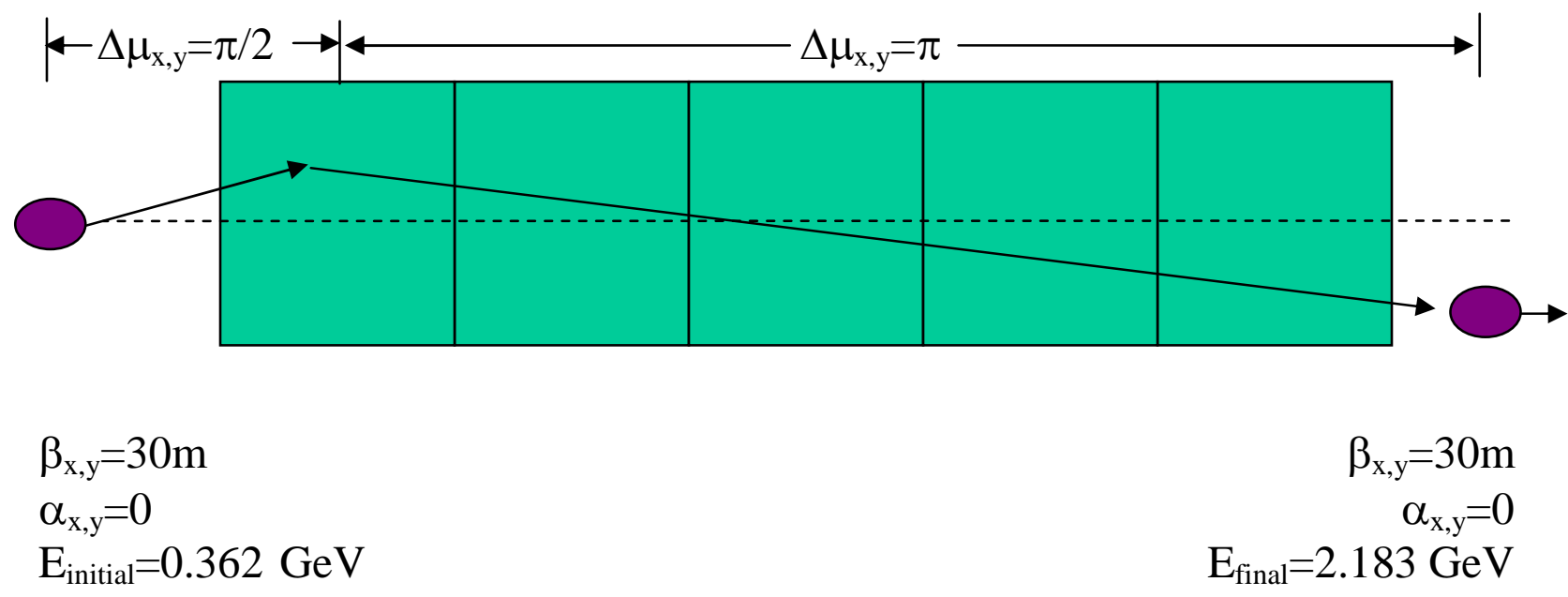

Figure 2: Schematic of the five resonator model showing the first bunch having an angular deviation prior to entering the first resonator. The two purple ovals represent the same bunch as it goes through a betatron phase advance of $3 \pi / 2$, entering with an angular deviation and leaving with an offset. The Twiss parameters at the starting and ending points are arbitrarily chosen, whereas the Twiss parameters inside the resonators correspond to actual Twiss parameters at the center of each resonator. 


\section{TESTING THE TRANSFER MATRIX}

We used the transfer matrix

$$
\mathrm{M}=\left[\begin{array}{cc}
\sqrt{\frac{\beta_{2}}{\beta_{1}}}\left(\cos \Delta \psi+\alpha_{1} \sin \Delta \psi\right) & \sqrt{\beta_{1} \beta_{2}} \sin \Delta \psi \\
-\frac{\left(1+\alpha_{1} \alpha_{2}\right) \sin \Delta \psi+\left(\alpha_{2}-\alpha_{1}\right) \cos \Delta \psi}{\sqrt{\beta_{1} \beta_{2}}} & \sqrt{\frac{\beta_{1}}{\beta_{2}}}\left(\cos \Delta \psi-\alpha_{2} \sin \Delta \psi\right)
\end{array}\right]
$$

to find the offset and angle in the first resonator (assuming that $\beta_{\mathrm{x}}, \beta_{\mathrm{y}}=30 \mathrm{~m}$ and $\alpha_{\mathrm{x}}, \alpha_{\mathrm{y}}=0$ at the starting point). In the matrix, $\beta_{1}$ is the beta-function at the initial point, $\beta_{2}$ is the beta-function at the final point, $\alpha_{1}$ is the alpha-function at the initial point, $\alpha_{2}$ is the alpha-function at the final point, and $\Delta \psi$ is the betatron phase difference between the initial and final points.

Prior to starting the wake field calculations, we decided to test the program which applies the transfer matrix to the initial offsets and angles and calculates the final offsets and angles. We chose an end point that has a $3 \pi / 2$ phase advance from the starting point. This way the transfer matrix program should take an initial angular deviation and turn it into an offset with no angle at the end. A plot of offset (in the x-direction) versus betatron phase advance along the linac is shown in Figure 3.

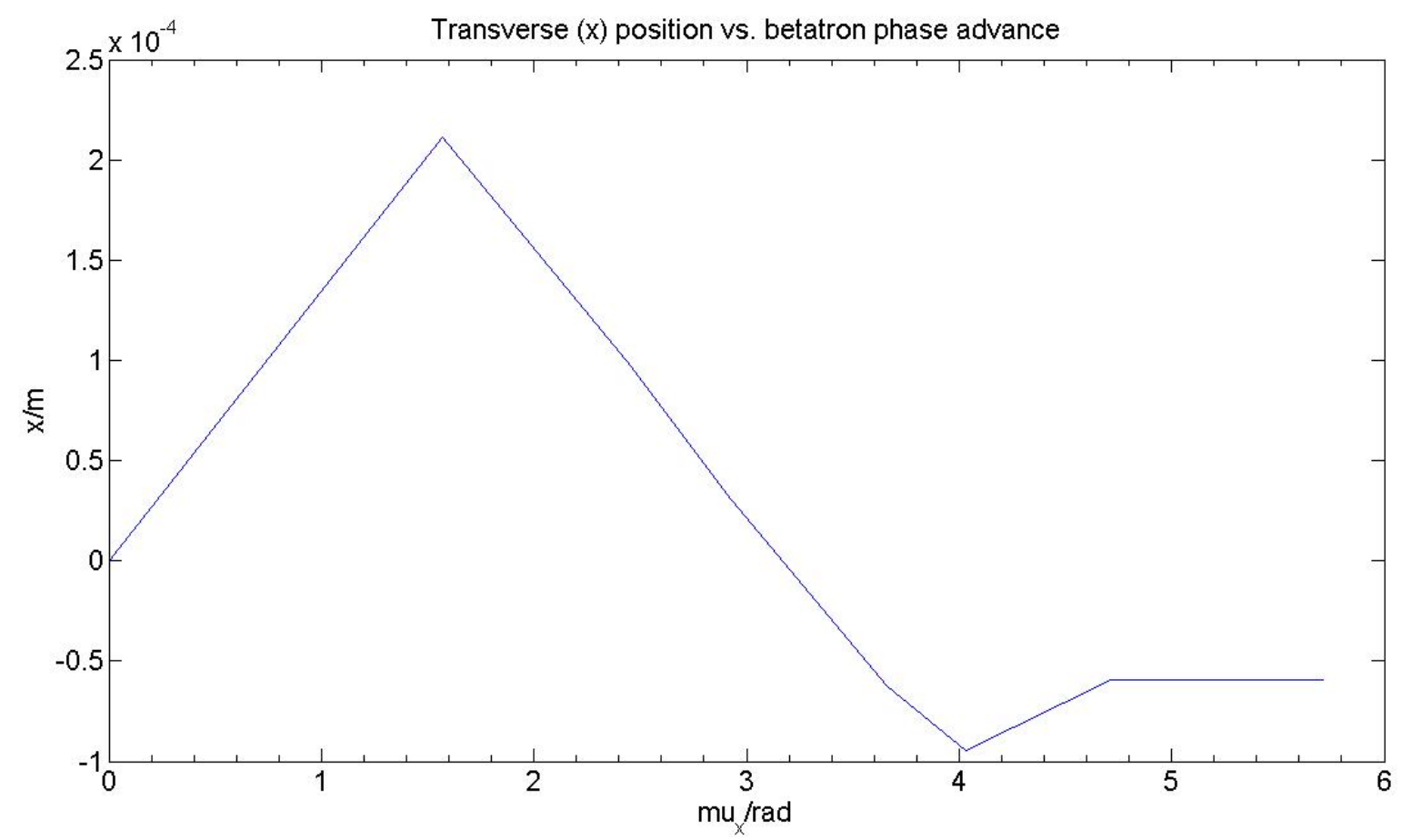

Figure 3: Transverse position of a bunch moving through the five resonators without encountering any wake fields. This shows that the transfer matrix program works since the bunch goes from no offset (with an angle) to no angle (but with an offset) during a phase advance of $3 \pi / 2$. 


\section{WAKE FIELDS IN THE FIVE RESONATOR MODEL}

Bunches following the off-angle bunch will experience a transverse momentum kick due to the wake field left behind by the off-angle bunch. When this kick occurs, they will obtain an angle (or an additional angle):

$$
\Delta(\mathrm{dx} / \mathrm{ds})=\Delta \mathrm{p}_{\perp} / \mathrm{p}_{0}
$$

where $\mathrm{p}_{0}$ is the longitudinal momentum of the beam, and $\Delta \mathrm{p}_{\perp}$ can be defined with the help of the single-bunch wake as:

$$
\Delta \mathrm{p}_{\perp}(\mathrm{t})=(1 / \mathrm{c}) \int_{\mathrm{S}_{0}}^{\mathrm{S}_{0}+\mathrm{L}} \int_{0}^{\infty} \mathrm{W}\left(\mathrm{t}-\mathrm{t}^{\prime}\right) \mathrm{q}\left(\mathrm{t}^{\prime}\right) \mathrm{x}\left(\mathrm{t}^{\prime}\right) d \mathrm{t}^{\prime} \mathrm{ds}
$$

where $\mathrm{W}$ is the wake function (Equation 1), $\mathrm{W}\left(\mathrm{t}-\mathrm{t}^{\prime}\right)=0$ for $\mathrm{t}\left\langle\mathrm{t}^{\prime}, \mathrm{q}\left(\mathrm{t}^{\prime}\right)\right.$ is magnitude of the charge in a cross-section of the beam at time $\mathrm{t}^{\prime}, \mathrm{x}\left(\mathrm{t}^{\prime}\right)$ is the transverse displacement of a slice of beam at time $\mathrm{t}^{\prime}, \mathrm{S}_{0}$ is some distance along the linac, and $\mathrm{L}$ is the length of a structure (usually a resonant cavity). In general, the wake field could depend upon the distance along the linac (in case the properties of the cavities changes), but in our linac, the integrand is independent of $s$, and so the double integral reduces to a single integral over $\mathrm{t}^{\prime}$, multiplied by L.

After calculating the angles in the first resonator, we apply the transfer matrix (using actual Twiss parameter values and energies for the center of each resonator) to find offsets and angles in the next resonator. We continue in this manner through the five resonators.

\section{RESULTS OF THE FIVE RESONATOR MODEL}

In order to make our results easier to see and understand, we pick our end point so that the total betatron phase advance from start to end is $3 \pi / 2$. We assume that the end point has the same Twiss parameter values as the starting point. From the definition of normalized emittance in the case where $\alpha_{\mathrm{x}}=0$,

$$
\varepsilon=x^{2} / \beta_{x}+\beta_{x}^{*}\left(x^{\prime}\right)^{2}
$$

we can define an effective beam size (really the amplitude of betatron oscillations):

$$
\sigma_{\text {eff }}=\sqrt{\beta^{*} \varepsilon}=\sqrt{\mathrm{x}^{2}+\left(\beta \mathrm{x}^{\prime}\right)^{2}}
$$

We then define gain as $\sigma_{\text {eff }}^{(n)} / \sigma_{\text {eff }}^{(0)}$ where $n$ is the index of the bunch $(n=0$ is the first bunch having an angular deviation). It is interesting to note that since the $0^{\text {th }}$ bunch starts with only an angular deviation of $\sigma^{\prime}$ and has a betatron phase advance of $3 \pi / 2$ through the linac, (having the same alpha and beta functions at the beginning and end) $\sigma_{\text {eff }}{ }^{(0)}$ is in fact the rms beam size at the end of the linac. Below are the results of the five resonator model with just one bunch starting with an angle (Figures 4 and 5). 


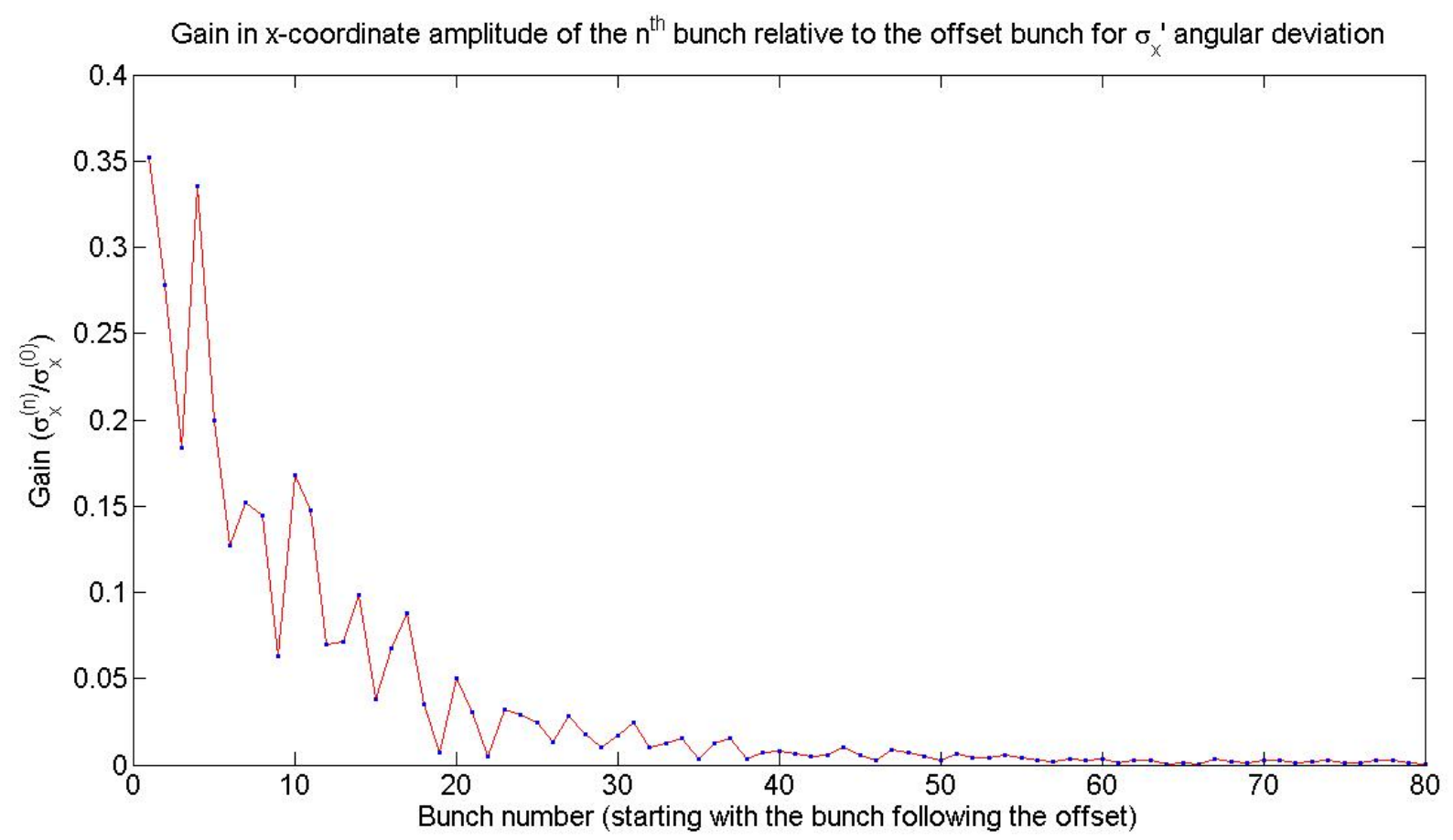

Figure 4: Plot of the amplitude of betatron oscillations at the end of the linac normalized by the amplitude of betatron oscillations of the off-angle bunch ( $\sigma_{x}$ at the end) when the first bunch starts with an angular deviation of $\sigma_{x}{ }^{\prime}$.

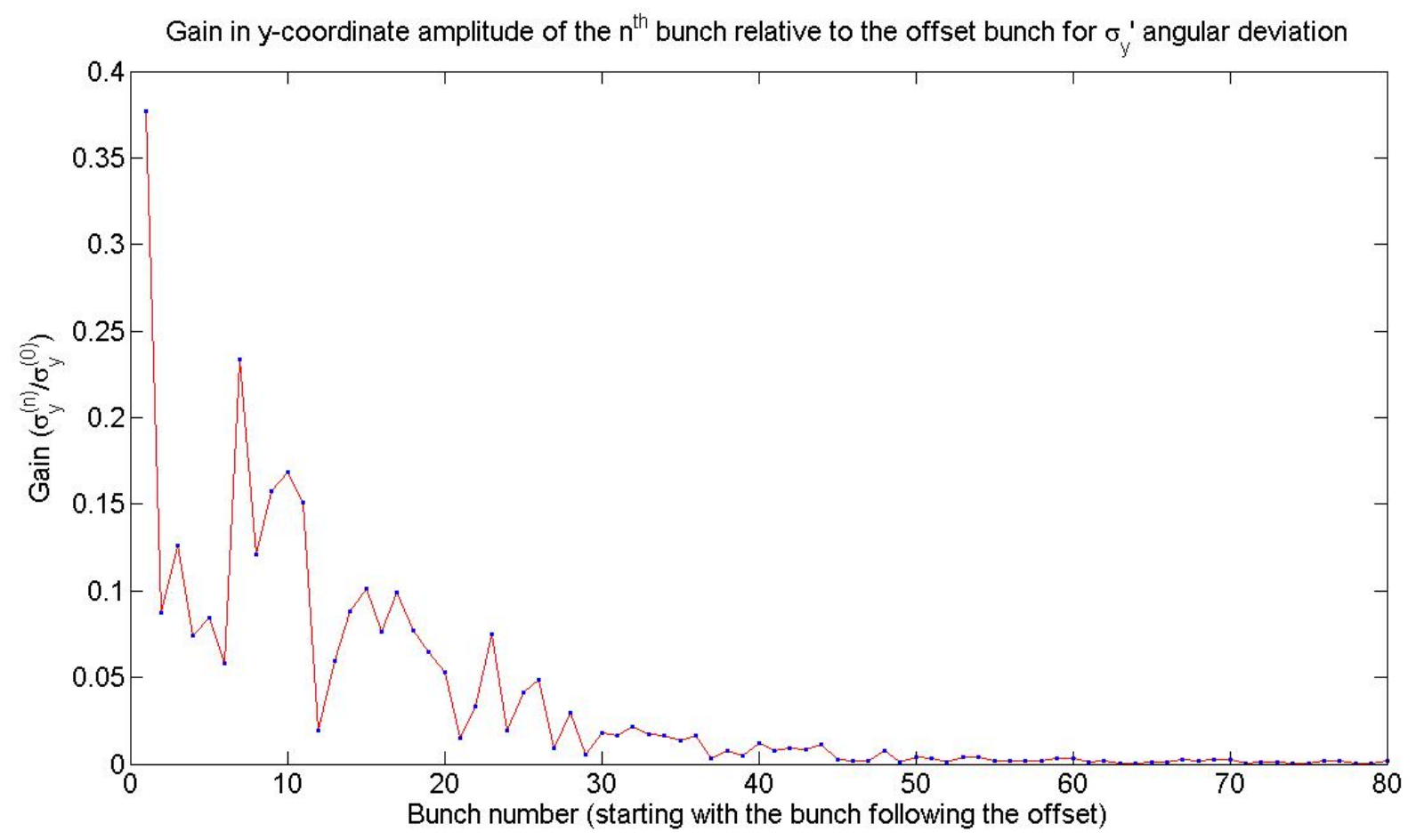

Figure 5: Plot of the amplitude of betatron oscillations at the end of the linac normalized by the amplitude of betatron oscillations of the off-angle bunch $\left(\sigma_{y}\right.$ at the end) when the first bunch starts with an angular deviation of $\sigma_{y}{ }^{\prime}$. 
We can see that the final amplitude of oscillation of a bunch following the off-angle bunch is only about $40 \%$ of the final amplitude of oscillation of the off-angle bunch. We can also see that amplitudes of oscillation decrease along the bunch train. This shows that the wake fields left behind by the bunches do not lead to an amplification of the initial deviation. The effects of the wake fields are relatively small, leading to a damping of beam instability in the case where one bunch begins on-axis with an angle.

We now continue our investigation by looking at a scenario where, after one bunch begins off-angle, all subsequent bunches have the same angular deviation. This will lead to a new steady-state trajectory at the end of the linac. Of course, if wake field effects are strong, the steady-state may not be reached due to beam break-up. We assume that bunches enter with an angular deviation of $\sigma_{\mathrm{x}}{ }^{\prime}$ (or $\sigma_{\mathrm{y}}{ }^{\prime}$ ) and check whether an instability occurs. The results are shown below in Figures 6 and 7.

Gain in $\mathrm{x}$-coordinate amplitude of the $\mathrm{n}^{\text {th }}$ bunch relative to the first bunch for $\sigma_{\mathrm{x}}{ }^{\prime}$ angular deviation of all bunches

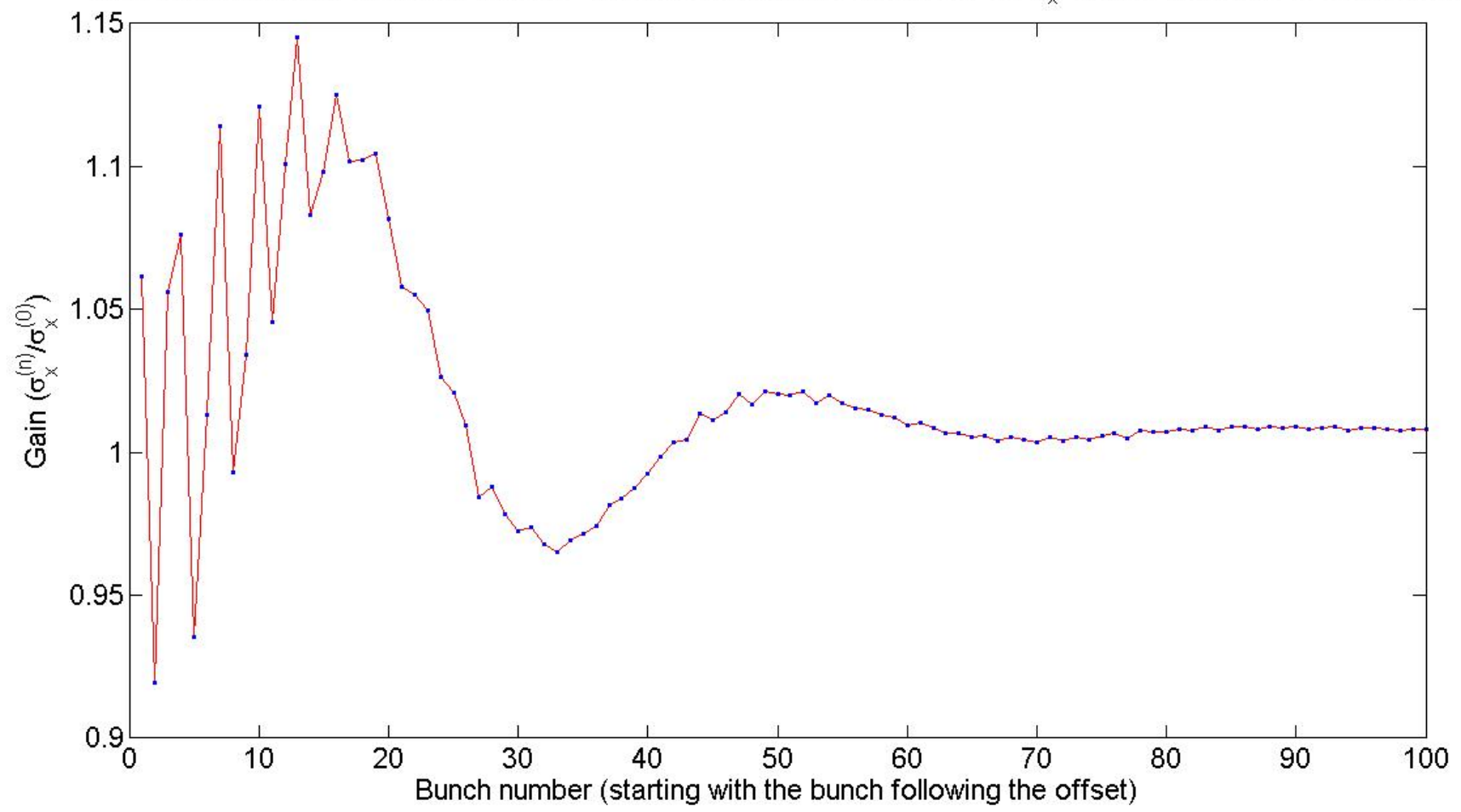

Figure 6: Plot of the amplitude of betatron oscillations at the end of the linac normalized by the amplitude of betatron oscillations of the first off-angle bunch $\left(\sigma_{x}\right.$ at the end) when the all bunches start with an angular deviation of $\sigma_{x}{ }^{\prime}$. 


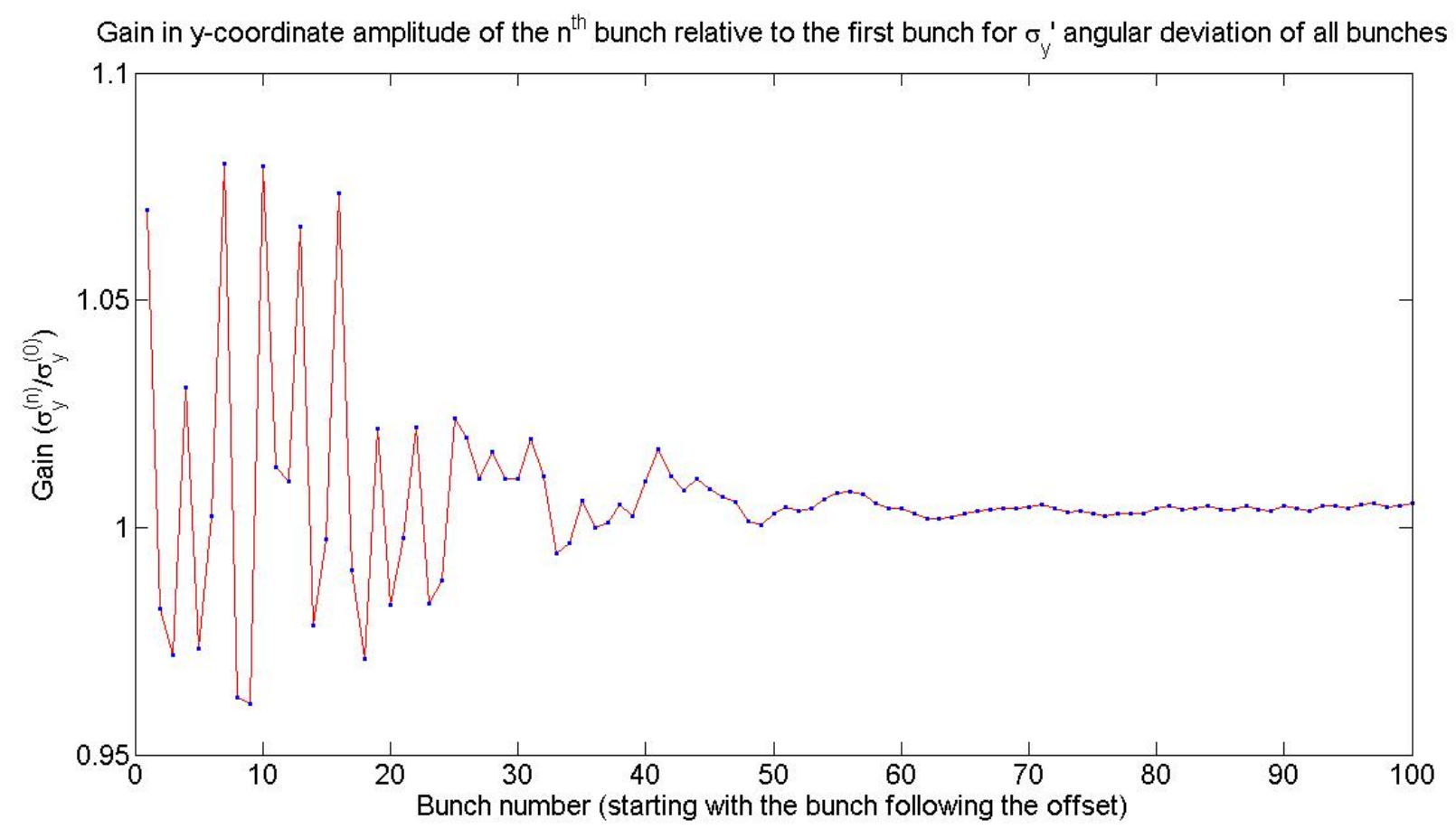

Figure 7: Plot of the amplitude of betatron oscillations at the end of the linac normalized by the amplitude of betatron oscillations of the first off-angle bunch ( $\sigma_{y}$ at the end) when the all bunches start with an angular deviation of $\sigma_{y}^{\prime}$.

We see that the new steady-state trajectory is reached after only a short transient regime where bunches oscillate about the new steady-state trajectory. The amplitudes of oscillation are not great even in the transient regime, on the order of about $10 \%$ of the difference between the initial and final steady-state trajectories.

Given the results of the two previous cases, it seems that there will not be any beam instability in the BFLS. We will check this in the case when the initial angular deviation is given by a sinusoidal function:

$$
\mathrm{x}=\sigma_{\mathrm{x}}{ }^{\prime} \sin (\omega \mathrm{t})
$$

This could happen if some unstable element prior to the five resonator cavities has a jitter which kicks the bunches up and down with some periodicity. We assume that such an element has a period that is long compare to the bunch spacing, and as a specific example, we took a modulation frequency of $10 \mathrm{kHz}$ in order to observe the results. We want to be sure that there is no instability in this situation, since such a jitter could occur in the BFLS. The results are plotted in Figures 8 and 9. 
Amplitude of $\mathrm{n}^{\text {th }}$ bunch normalized by final $\sigma_{x}$ with angular deviation given by a sine function with amplitude $\sigma_{x}{ }^{\prime}$ and $\omega=6.28^{\star} 10^{4}$

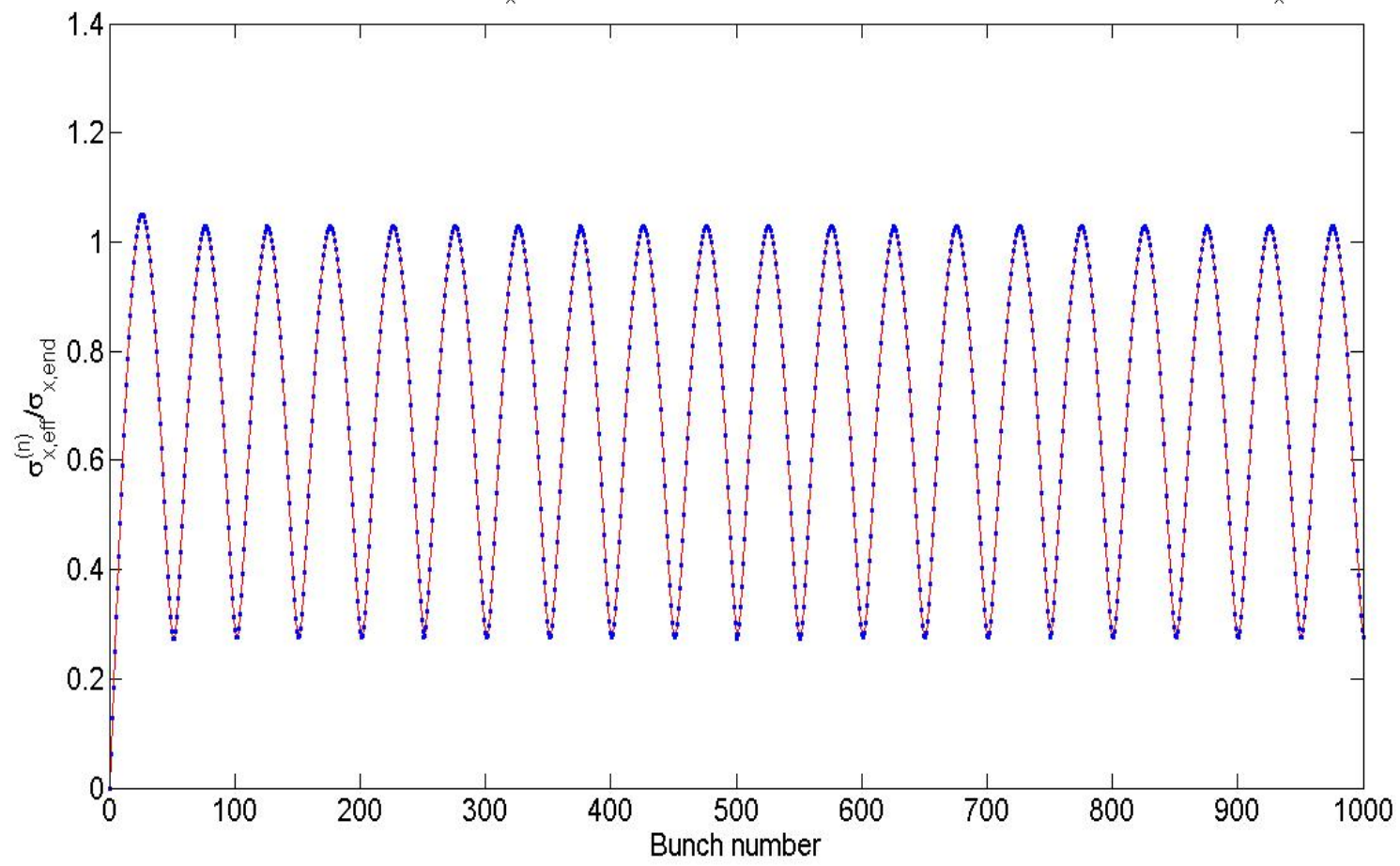

Figure 8: Plot of how the bunch train responds to an element that causes bunches to have a sinusoidal variation in initial offsets (in the $x$-direction).

Amplitude of $\mathrm{n}^{\text {th }}$ bunch normalized by final $\sigma_{y}$ with angular deviation given by a sine function with amplitude $\sigma_{y}{ }^{\prime}$ and $\omega=6.28^{*} 10^{4}$

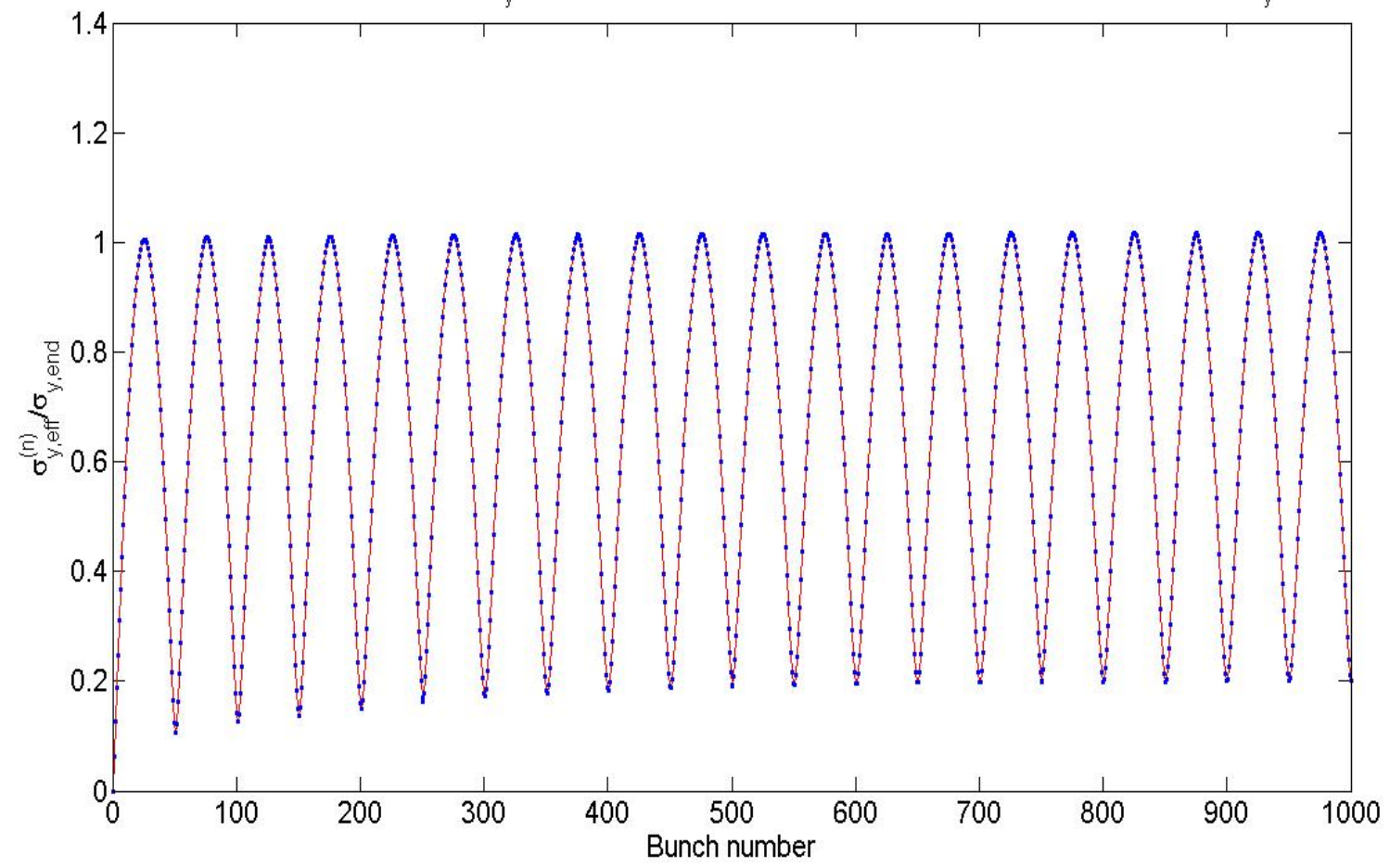

Figure 9: Plot of how the bunch train responds to an element that causes bunches to have a sinusoidal variation in initial offsets (in the y-direction). 
The beam's response to an element with a jitter causing a sinusoidal variation in initial angular deviation confirms that the wake fields in the BFLS do not lead to beam instability. The bunches essentially follow the trajectories that their angular deviations give them, except that bunches having almost no initial angle are moved off-axis to about a fifth of the amplitude of the sine wave. This is not an issue, as long as the maximum amplitude of oscillations at the end of the linac is not greatly increased by the wake fields. This is exactly the case, as bunches entering with the maximum angular deviation $\sigma_{\mathrm{x}}{ }^{\prime}\left(\sigma_{\mathrm{y}}{ }^{\prime}\right)$ exit the linac with an amplitude of oscillation of about $\sigma_{\mathrm{x}}\left(\sigma_{\mathrm{y}}\right)$, meaning that the wake fields do not cause their trajectories to deviate any further from the axis of the linac.

\section{EXPLORATORY STUDY}

We are interested in finding out how close the Q-values of the various higher-order modes are to causing beam break-up. Higher Q-values would result in the wake field not being damped as quickly, leading to a greater transverse momentum kick to subsequent bunches.

We proceed with a trial-and-error method, multiplying the Q-values of all the modes until a gain of about 10 is reached. We consider a gain of 10 to be unstable because we assume that bunches in the linac will deviate (initially) by no more than $0.1 \sigma$, and a final deviation of $\sigma$ could be large enough to cause problems in the free-electron laser, downstream of the linac. The results shown in Figure 10 were obtained for Q-values multiplied by 20 . One bunch moves through the linac off-axis, causing subsequent bunches to leave the axis. After about 200 bunches, a gain of 10 is reached, and is maintained for a large number of bunches $(\sim 350)$.

Further analysis showed that it is not necessary to multiply all Q-values by 20 . If just the mode with the highest loss factor has a higher Q-value than expected, beam break-up could occur. In fact, we found that the mode with frequency $2.58 \mathrm{GHz}$ is the most important in determining beam break-up. Multiplying the Q-value of only that mode by 20 yields results that are similar to multiplying the Qvalues of all modes by 20 (see Figure 11). It appears that other modes are not very important in determining beam break up. Figure 12 shows what happens when their Q-values are multiplied by 20 , while the Q-value of the mode with the highest loss factor is unchanged. 


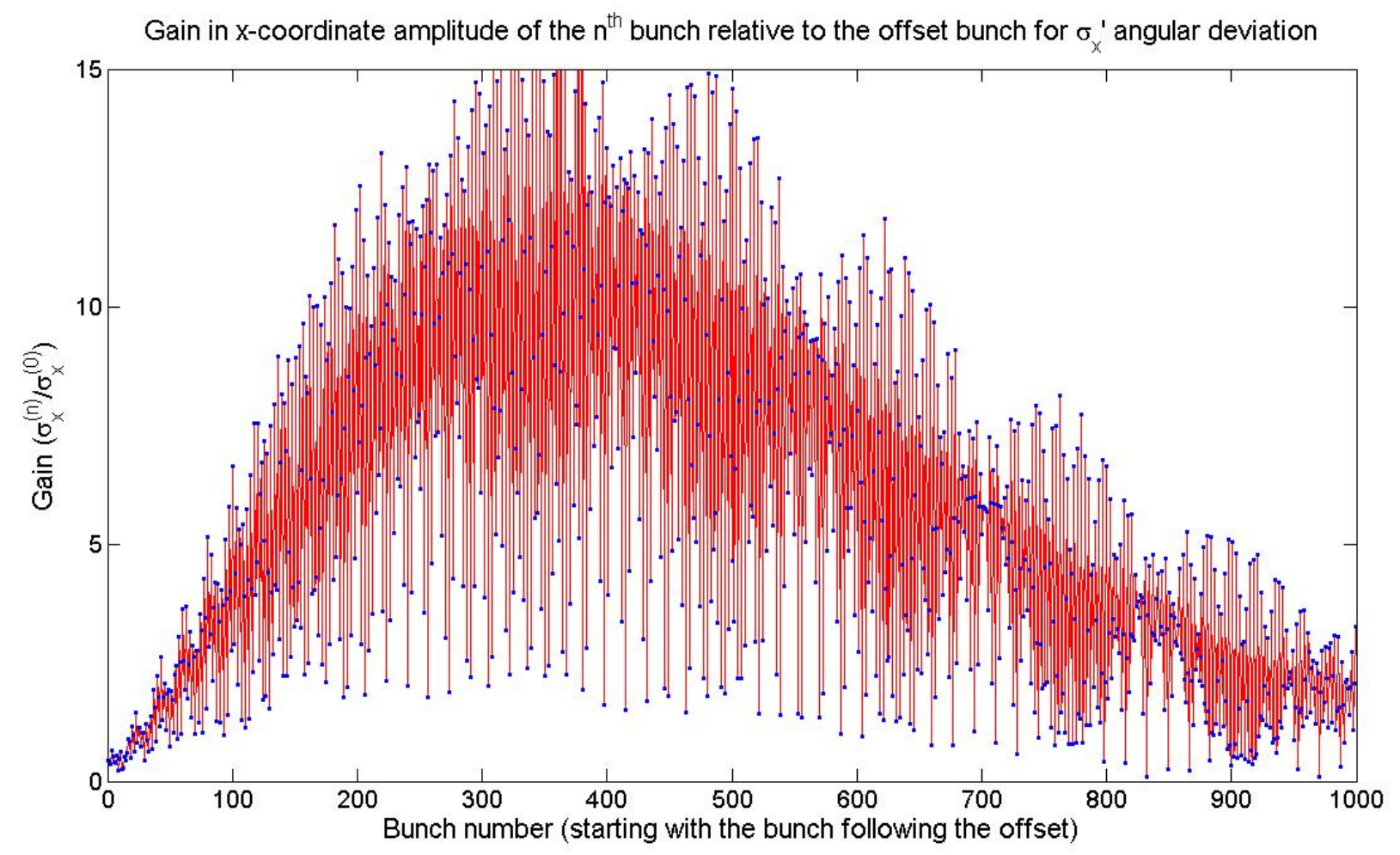

Figure 10: Plot of the amplitude of betatron oscillations of each bunch at the end of the linac after a bunch (numbered 0 ) starts with an angular deviation of $\sigma_{x}{ }^{\prime}$. The $Q$-values for each of the higher-order modes are 20 times greater here than when a similar situation was considered in Figure 4.

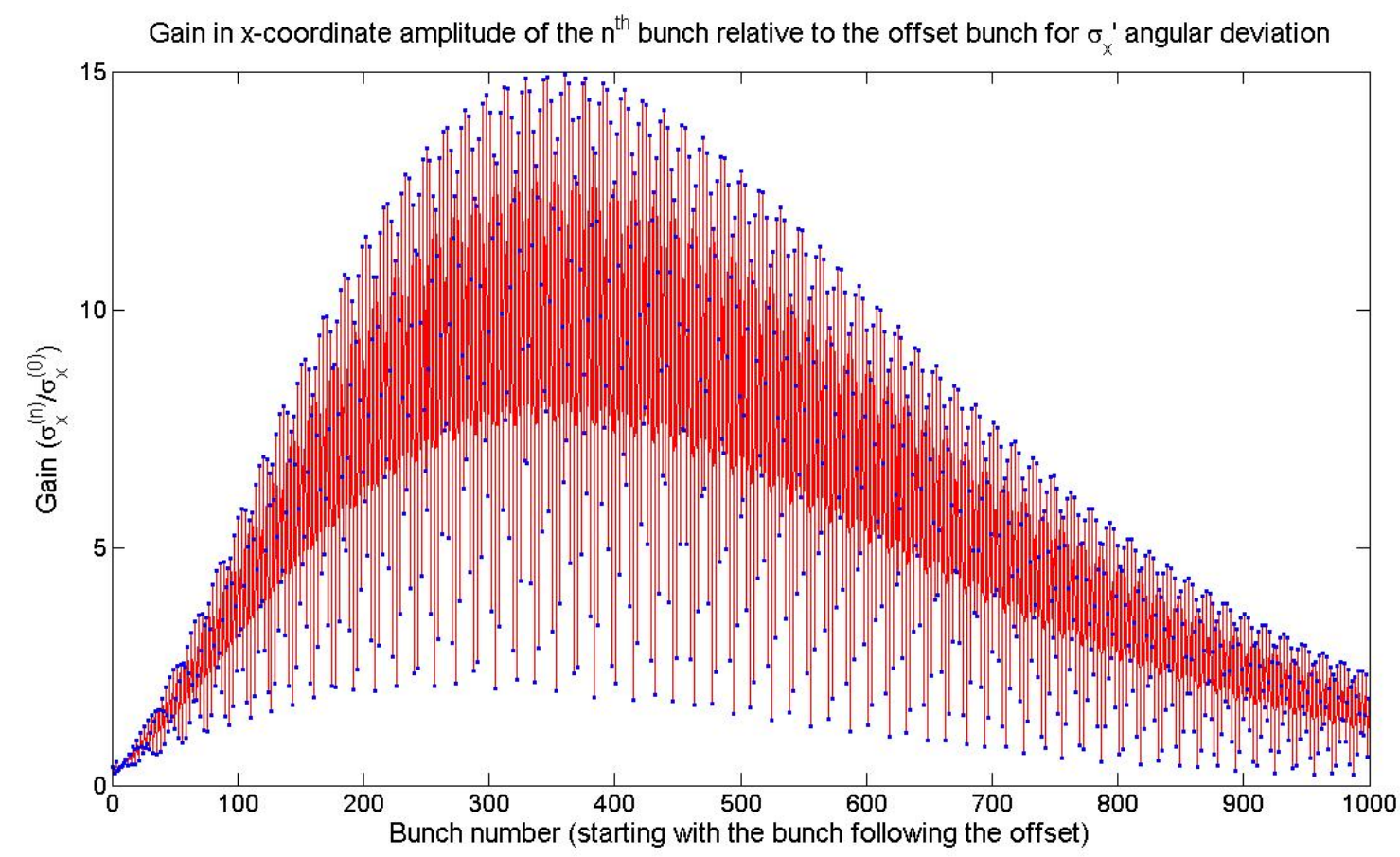

Figure 11: Plot of the amplitude of betatron oscillations at the end of the linac as in Figure 10. Here, only the $Q$-value of the mode with the highest loss factor (frequency of $2.58 \mathrm{GHz}$ ) is multiplied by 20. 


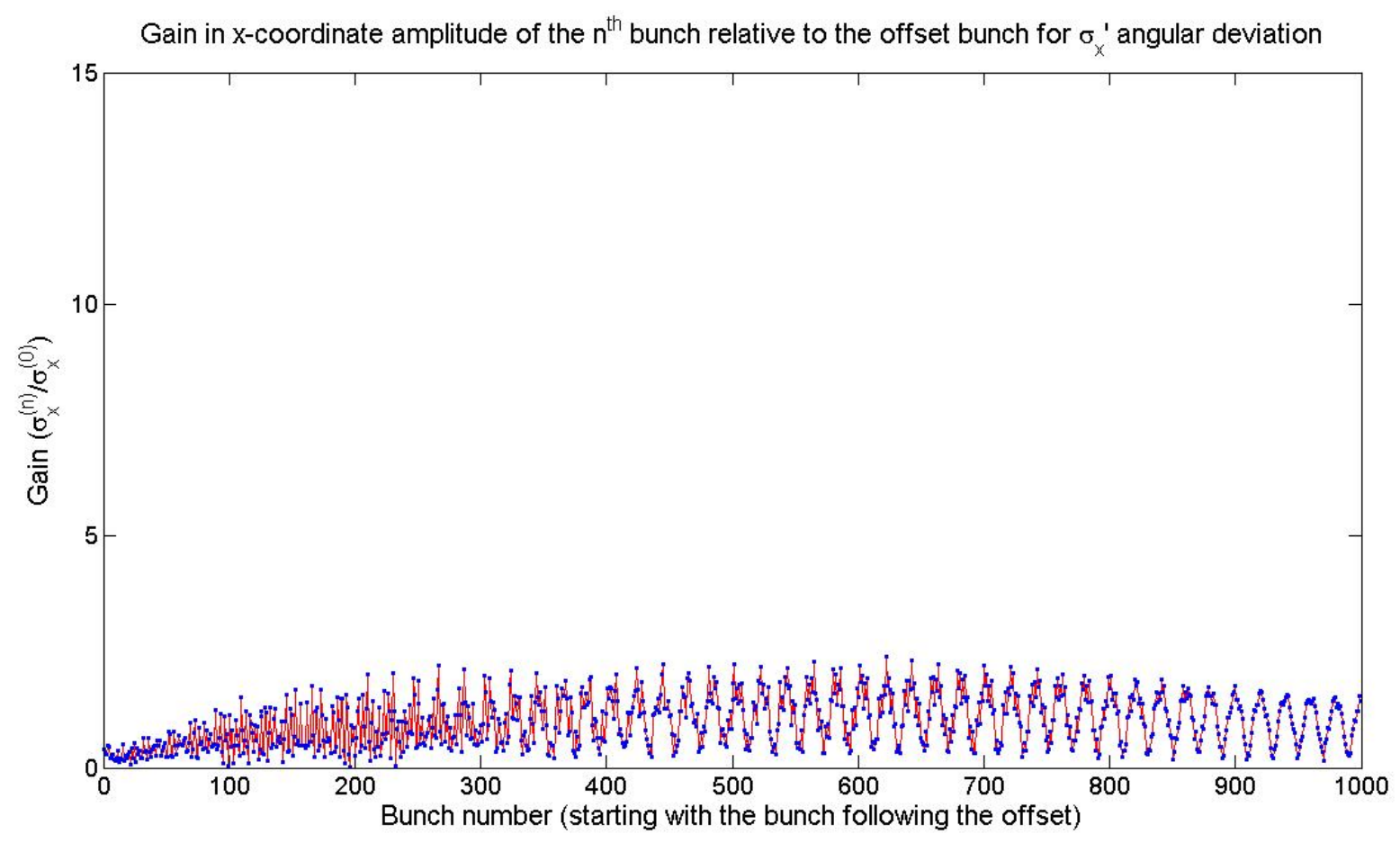

Figure 12: Plot of the amplitude of betatron oscillations as in Figure 10, but the $Q$-value of the mode with the highest loss factor is not multiplied by 20.

\section{CONCLUSIONS}

We can generalize the results of our simulations to the case where each bunch follows an orbit that does not coincide with the axis of the linac. A single bunch deviating from a long-established orbit will not cause a runaway instability. Instead, its effects on subsequent bunches become damped along the bunch train. If a constant injection error occurs after some time, (i.e. after an orbit has been established, some bunch and all subsequent bunches deviate from it) the beam will move to a new orbit after a very short transient regime. In the transient regime, the bunches will oscillate about the new orbit, but with a small amplitude compared to the distance between the two orbits. Finally, if there is an injection error that has a periodic nature (some unstable element causes bunches to have sinusoidal variation of either angles or offsets) the beam will follow the instability of that element, without too much being added by wake field effects. The findings suggest that multi-bunch beam break-up due to long-range transverse wake fields will not be an issue in the BFLS. 
Acknowledgement. Work supported by Department of Energy Contract No. DE-AC02-05CH11231.

\section{REFERENCES}

1. A. Mosnier, Instabilities in Linacs, CEA Saclay internal rep. DAPNIA/SEA 93-19, December 1993

2. TESLA Design Report, DESY

3. N. Baboi, Studies on Higher Order Modes in Accelerating Structures for Linear Colliders, Hamburg, April 2001 